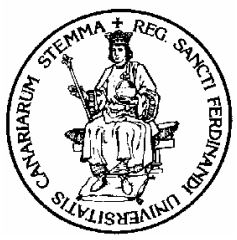

Fac. CC. Económicas y Empresariales Universidad de La Laguna
Fac. CC. Económicas y Empresariales Univ. de Las Palmas de Gran Canaria

\title{
New nonlinear approaches for the adjustment and updating of a SAM
}

\author{
Casiano Manrique de Lara Peñate* \\ Dolores R. Santos Peñate **
}

DOCUMENTO DE TRABAJO 2003-07

* Universidad de Las Palmas de Gran Canaria. Departamento de Análisis Económico Aplicado.

** Universidad de Las Palmas de Gran Canaria. Departamento de Métodos Cuantitativos en Economía y Gestión. 
New Nonlinear Approaches for the Adjustment and Updating of a SAM

Manrique de Lara Peñate, Casiano

Santos Peñate, Dolores

University of Las Palmas de G.C.; Saulo Torón, 4;

Departamento de Análisis Económico Aplicado ; Edificio D-2-21

35017 Las Palmas de G.C.; SPAIN

casiano@empresariales.ulpgc.es

JEL :

C61 - Optimization Techniques; Programming Models;

Dynamic Analysis

C67 - Input-Output Models 
New Nonlinear Approaches for the Adjustment and Updating of a SAM

\begin{abstract}
We believe that any adjustment and updating process (AUP) should try to minimize the relative deviation of the new coefficients from the initial ones in a homogeneous way. This homogeneity would mean that the magnitude of this relative deviation is similar among the elements of each row or column, therefore avoiding the concentration of the changes in particular cells of the SAM.

In this work, we propose some new adjustment criteria in order to obtain a homogeneous relative adjustment of the structural coefficients. We also test the usefulness of this proposal by comparing its results with the ones obtained by more standard approaches.
\end{abstract}

\title{
Resumen
}

En nuestra opinión, todo proceso de ajuste y actualización debe procurar minimizar las desviaciones relativas entre los nuevos coeficientes y los iniciales de la forma más homogénea posible. Esta homogeneidad haría que la magnitud de la desviación relativa fuera similar a lo largo de los elementos de una columna o fila, evitando así que los cambios en los coeficientes se concentren en celdas particulares de la Matriz de Contabilidad Social.

En este trabajo, proponemos nuevos enfoques de ajuste que permiten obtener un ajuste más homogéneo de los coeficientes estructurales. Igualmente comparamos la utilidad de nuestra propuesta con métodos más estándar. 


\section{Introduction}

Many structural relations should be taken into account in any reasonable adjustment and updating process (AUP) of a social accounting matrix (SAM). These structural relations are mainly represented by ratios of different types such as technical coefficients or the proportion of the value of a cell in relation to its row or column total. When the AUP has as one of its aims the preservation of the initially observed structural relations, the procedure to be employed has to be able to maintain these technical, row or column coefficients as close as possible to the ones used as a starting point, avoiding the concentration of the changes in the coefficients in particular cells of the SAM.

This is the case when the time elapsed since the estimation of the SAM is not long enough to allow for any significant structural change. In these cases we believe that any updating process should try to minimize the relative deviation of the new coefficients from the initial ones in a homogeneous way. This homogeneity would mean that the magnitude of this relative deviation is similar among the elements of each row or column.

On the other hand, most practical efforts to update SAMs would generate very complicated nonlinear programs for which even obtaining a solution could prove to be very difficult, especially when updating very disaggregated accounts. This is especially the case when we introduce more than one coefficient in the objective function (e.g.: technical coefficients, row and column coefficients or some combination of all three). In many occasions this forces practitioners to introduce exogenous bounds to the different elements of the SAM matrix that bias the results in an artificial manner.

This study has two main objectives. First, we propose different formulations that try to obtain a more homogeneous relative adjustment of the structural coefficients while reducing the non-linearity of the programs in order to ease obtaining a solution. These formulations combine the approach proposed by Matuszewski, Pitts and Sawyer (1964) with other adjustment criteria

Second, we try to test the usefulness of this proposal by comparing its results with the ones obtained by more standard approaches (RAS and the minimum sum of cross 
entropies criterion). We are able to show that these approaches tend to produce, under certain conditions, a less homogeneous pattern of coefficient adjustment than the ones we propose.

The following section presents a brief summary of the main contributions found in the literature about the adjustment of SAMs, using mathematical programming, that have been considered in the comparison exercises. Section three summarizes our approaches in mathematical terms. Finally the results of the different comparisons carried out to evaluate the usefulness of our approaches as well as a short section with our main conclusions are presented.

\section{Updating and Adjustment Criteria}

A Social Accounting Matrix (SAM) is a square matrix $X$ of order $n$ whose rows and columns represent separate accounts for which expenditures (columns) and receipts (rows) must balance. This balance can be expressed mathematically as follows:

$$
\sum_{k} x_{i k}=\sum_{k} x_{k i}, \quad \forall i
$$

where the element $x_{i j}$ denotes the payments of account $\mathrm{j}$ to account $\mathrm{i}$. Associated with each SAM we can define the following column coefficient matrix $B=\left(b_{i j}\right)_{1 \leq i, j \leq n}$ as

$$
b_{i j}=\frac{x_{i j}}{\sum_{i} x_{i j}}
$$

If $\sum_{k} x_{k j}=\gamma_{j}, j=1,2, \ldots, n$, the balance equation can also be expressed in terms of the column coefficients as

$$
\begin{gathered}
\sum_{j} b_{i j} \gamma_{j}=\gamma_{i}, \quad \forall i \\
\sum_{i} b_{i j}=1, \quad \forall j .
\end{gathered}
$$

Assuming that a previous column coefficient matrix $B^{0}$ (in time $\mathrm{T}=0$ ) and the sum of the elements of the columns, $\gamma_{j}^{t}>0, \forall j$, of the same SAM in time $\mathrm{t}$ are known, the adjusting and updating process (AUP) consists of defining a new matrix $B^{t}$ that 
satisfies conditions (2) and (3). To this end we apply an adjustment criterion that involves a dissimilarity measure between matrices.

We now describe the main characteristics of the two approaches considered in this paper as a benchmark for the comparisons with our proposals; the RAS and the minimum sum of cross entropies (MSCE) criterion. Both represent a classical and a very recent AUP respectively, that are very closely related to each other. Moreover we summarize the main characteristics of the approach proposed by Matuszewski, Pitts and Sawyer (1964) which serves as basis of our proposals.

\subsection{The RAS and the MSCE criteria}

Traditionally, the AUP has been solved using the RAS method which consists of obtaining a matrix $B^{t}$ that meets balance conditions (2) and (3), such that

$$
B^{t}=R B^{0} S
$$

where $\mathrm{R}$ and $\mathrm{S}$ are diagonal matrices of order $\mathrm{n}$ whose elements are all non null. That is, $R=\operatorname{diag}\left(r_{1}, r_{2}, \ldots, r_{n}\right), S=\operatorname{diag}\left(s_{1}, s_{2}, \ldots, s_{n}\right)$, and $b_{i j}^{t}=r_{i} b_{i j}^{0} s_{j}, \forall i, j$. Terms $\mathrm{r}$ and $\mathrm{s}$ can be given economic interpretations but necessarily incorporate the assumption that the effects identified are all uniform across sectors.

Bacharach (1965) extended this matrix adjustment criteria, altering the initial RAS approach stated by Stone (1962). He formulates a new problem, the biproportional adjustment problem, which consists of searching for a matrix $B=\left(b_{i j}\right)_{i, j}$ that satisfies the balance conditions (2) and (3), such that

$$
\begin{gathered}
b_{i j}^{t} \geq 0, \quad \forall i, j \\
B^{t}=\lim _{q \rightarrow \infty} R^{q} B^{0} S^{q}
\end{gathered}
$$


where $\left\{R^{q}\right\}$ and $\left\{S^{q}\right\}$ are sequences of diagonal matrices of order $\mathrm{n}$. A solution to this problem that can be expressed in the RAS form is called an interior solution, the rest are called frontier or contour solutions.

It has been proved (Bacharach, 1965; Macgill, 1977) that, if there exists a solution to the biproportional adjustment problem, it is unique. Also, if for each $x_{i j}$ non null $\gamma_{i} \leq \sum_{k \neq i} \gamma_{k}$ is verified, then the RAS method converges and obtains $B^{t}$ as its solution. Evidently, the RAS solution verifies that $b_{i j}^{t}=0 \Leftrightarrow b_{i j}^{0}=0$.

The minimum sum of cross entropies (MSCE) criteria (Golan, Judge and Robinson, 1994; McDougall, 1999; Robinson, Cattaneo and Moataz El-Said, 2000) consists of solving the following problem

$$
\min \sum_{(i, j) / b_{i j}^{0} \neq 0} b_{i j} \ln \frac{b_{i j}}{b_{i j}^{0}} \quad \text { subject to (2) and (3). }
$$

The objective function in (4) is the sum of the cross entropies corresponding to the columns of matrices $B$ and $B^{0}$. If we interpret each column $\mathrm{j}$ of matrices $B$ and $B^{0}$ as the a priori probability distribution, the cross entropy for $b_{j}=\left(b_{i j}\right)_{i}$ and $b_{j}^{0}=\left(b_{i j}^{0}\right)_{i}$, where $b_{j}^{0}$ represents the a priori knowledge, is given by

$$
\sum_{i / b_{i j}^{0} \neq 0} b_{i j} \ln \frac{b_{i j}}{b_{i j}^{0}}=\sum_{i / b_{i j}^{0} \neq 0} b_{i j} \ln b_{i j}-\sum_{i / b_{i j}^{0} \neq 0} b_{i j} \ln b_{i j}^{0}
$$

that is, the difference between the expected values, $\mathrm{E}\left(\ln b_{j}\right)-\mathrm{E}\left(\ln b_{j}^{0}\right)$, which is considered to be a discrimination measure between $b_{j}$ and $b_{j}^{0}$ (Golan, Judge and Miller, 1996). Both the RAS and the MSCE are specific cases of the weighted minimum sum of cross entropies (WMSCE) criterion, which consists of solving the following problem 


$$
\min \sum_{(i, j) / b_{i j}^{0} \neq 0} w_{j} b_{i j} \ln \frac{b_{i j}}{b_{i j}^{0}} \quad \text { subject to (2) and (3) }
$$

where $w_{j}$ are the weights used. The MSCE criteria can be derived by making $w_{j}=1, \forall j$ in the WMSCE formulation. The RAS method is actually an algorithm to solve the WMSCE criteria when

$$
w_{j}=\frac{\gamma_{j}^{t}}{\gamma^{t}}
$$

where $\gamma^{t}=\sum_{i j} x_{i j}^{t}=\sum_{j} \gamma_{j}^{t}$ (McDougall, 1999). This relationship between the RAS and the WMSCE criteria has also been demonstrated in the past (Macgill, 1977). Extending the results obtained by McDougall (1999) to the WMSCE the optimal solution to this problem is the following one:

$$
b_{i j}=\frac{1}{\Omega_{j}} b_{i j}^{0} e^{-\frac{\mu_{i} \gamma_{j}}{w_{j}}}, \forall i, j
$$

where

$$
\Omega_{j}=\frac{1}{\sum_{k} b_{k j}^{0} e^{-\frac{\mu_{k} \gamma_{j}}{w_{j}}}} .
$$

This expression is a result of the Lagrange optimality conditions for problem (5). The values $\mu_{\mathrm{i}}$ are the multipliers associated to constraints (2).

Operating with the definition of a column coefficient we can derive that the relationships of the previous proposition also apply when we substitute the coefficients $b_{j}$ and $b_{j}^{0}$ by the matrix terms $x_{i j}$ and $x_{i j}^{0}$ respectively.

\section{2.- Adjustment techniques defined as mathematical programmes}


Matuszewski, Pitts and Sawyer (1964) were the first to propose an adjustment technique expressed as linear programs. Their problem was formulated as follows:

$$
\min \sum_{(i, j) / b_{i j}^{0} \neq 0}\left|\frac{b_{i j}^{t}}{b_{i j}^{0}}-1\right|
$$

subject to:

$$
\begin{gathered}
\sum_{i / b_{i j}^{0} \neq 0} b_{i j}^{t} p_{j}^{t}=\gamma_{j}^{t} \\
\sum_{j / b_{i j}^{0} \neq 0} b_{i j}^{t} p_{j}^{t}=\rho_{i}^{t} \\
\frac{1}{2} \leq \frac{b_{i j}^{t}}{b_{i j}^{0}} \leq 2 \quad \forall(\mathrm{i}, \mathrm{j}) / b_{i j}^{0} \neq 0
\end{gathered}
$$

being:

$$
\begin{aligned}
& b_{i j}{ }^{0}: \text { technical coefficient matrix obtained from } X^{0} \\
& b_{i j}{ }^{t}: \text { technical coefficient matrix obtained from } X^{t} \\
& p_{j}{ }^{0}: \text { vector of effective production in } 0 \\
& p_{j}{ }^{t}: \text { vector of effective production in } \mathrm{t} \\
& \gamma_{j}{ }^{t}: \text { sum of the elements of column } \mathrm{j} \\
& \rho_{i}^{t}: \text { sum of the elements of row } \mathrm{i}
\end{aligned}
$$

Their last group of restrictions were introduced to avoid the fact that the changes in the coefficients tended to concentrate in the larger elements of the intermediate transaction matrix. It is clearly arbitrary but it helped to increase the number of basic variables thus giving more realistic solutions.

Since the new vector of production was known to Matuszewski, Pitts and Sawyer (1964), they switched from using coefficients to flows taking the inverse of the new known values of effective production as weights. They converted this nonlinear formulation into a linear one by including two new positive variables for each of the elements to be updated, avoiding the existing nonlinearity in the objective function due to the calculation of absolute values. 
This need to set bounds to the variables is present in many other examples. From the more open formulations of Harrigan and Buchanan (1984) to the ones proposed by Zenios, Drud and Mulvay (1989) and Schneider and Zenios (1990). In fact the need of these bounds in twofold. First, it helps the programing solver to find a solution, and second, it helps to avoid corner solutions that are too extreme. However, once we impose these restrictions it is very easy to remain at the minimum or maximum values imposed, thus reducing the freedom to find the optimal solution. Our proposal shows there are alternative ways to find new coefficients without imposing such strong restrictions on the updating process.

\section{The models proposed}

In this section we will give account of the notation, definitions and adjustment criteria needed to describe the AUP models proposed in this paper, followed by a presentation of the specific formulations used.

\subsection{Notation, definitions and adjustment criteria}

Being $X=\left(x_{i j}\right)_{1 \leq i \leq m, 1 \leq j \leq n}$, we consider the following sets, matrices and functions.

\section{$\underline{\text { SETS: }}$}




$$
\begin{aligned}
& I=\{1,2, \ldots, m\} \\
& J=\{1,2, \ldots, n\} \\
& I_{+}=\left\{i \in I / \sum_{k} x_{i k} \neq 0\right\} \\
& J_{+}=\left\{j \in J / \sum_{k} x_{k j} \neq 0\right\} \\
& I_{j}=\left\{i \in I / x_{i j} \neq 0\right\} \text { and } n_{j}=\left|I_{j}\right| \text { is the cardinal of set } I_{j}, \forall j \in J \\
& J_{i}=\left\{j \in J / x_{i j} \neq 0\right\} \text { and } \mathrm{m}_{i}=\left|J_{j}\right| \text { is the cardinal of set } \mathrm{J}_{i}, \forall i \in I \\
& S_{x}=\left\{(i, j) / x_{i j} \neq 0, i \in I, j \in J\right\}
\end{aligned}
$$

\section{MATRICES:}

Row coefficient matrix, $A_{x}=\left(a_{i j}\right)_{1 \leq i \leq m, 1 \leq j \leq n}$ :

$$
a_{i j}:\left\{\begin{array}{lll}
\frac{x_{i j}}{\sum_{k} x_{i k}} & \text { if } & \sum_{k} x_{i k} \neq 0 \\
0 & \text { otherwise }
\end{array}\right.
$$

Column coefficient matrix, $B_{x}=\left(b_{i j}\right)_{1 \leq i \leq m, 1 \leq j \leq n}$ :

$$
b_{i j}:\left\{\begin{array}{ccc}
\frac{x_{i j}}{\sum_{k} x_{k j}} & \text { if } & \sum_{k} x_{k j} \neq 0 \\
0 & \text { otherwise }
\end{array}\right.
$$

Note that $S_{X}=S_{A_{X}}=S_{B_{X}}$.

\section{FUNCTIONS:}

Given the $m \times n$ matrix $\bar{X}=\left(\bar{x}_{i j}\right)$ with $S_{X}=S_{\bar{X}}$, we define

$$
\begin{aligned}
& F_{1}(X)=\sum_{(i, j) \in S_{x}}\left|\frac{x_{i j}-\bar{x}_{i j}}{\bar{x}_{i j}}\right| \\
& F_{2}(X)=\sum_{(i, j) \in S_{x}}\left|\frac{x_{i j}}{\bar{x}_{i j}}-\mu_{i}\right| \quad \text { where } \quad \mu_{i}=\frac{1}{m_{i}} \sum_{j \in J_{i}} \frac{x_{i j}}{\bar{x}_{i j}} \\
& F_{3}(X)=\sum_{(i, j) \in S_{x}}\left|\frac{x_{i j}}{\bar{x}_{i j}}-v_{j}\right| \quad \text { where } \quad v_{j}=\frac{1}{n_{j}} \sum_{i \in I_{j}} \frac{x_{i j}}{\bar{x}_{i j}}
\end{aligned}
$$




$$
\begin{aligned}
& F_{4}(X)=\sum_{(i, j) \in I \times(J-\{n\})}|| d_{i j}-1|-| d_{i j+1}-1|| \\
& \text { where } d_{i j}=\left\{\begin{array}{cc}
\frac{x_{i j}}{\bar{x}_{i j}} & \text { if } \quad(i, j) \in S_{x} \\
1 & \text { otherwise }
\end{array}\right. \\
& F_{5}(X)=\sum_{(i, j) \in(I-\{m\}) \times J}|| d_{i j}-1|-| d_{i+1 j}-1|| \\
& F_{6}(X)=\max _{i j}\left|\frac{x_{i j}-\bar{x}_{i j} \mid}{\bar{x}_{i j}}\right|
\end{aligned}
$$

Given the matrix $X^{0}=\left(x_{i j}^{0}\right)_{1 \leq i \leq m, 1 \leq j \leq n}$, the adjustment problem is to determine a matrix $X^{t}=\left(x_{i j}^{t}\right)_{1 \leq i \leq m, 1 \leq j \leq n}$ with a structure similar to $X^{0}$ satisfying certain constraints. The problem is formulated as an optimization problem where the objective function is a linear combination of the $F_{i}$ functions applied to particular coefficient matrices, replacing $\bar{X}$ by the corresponding matrix when $t=0$. We now consider the following adjustment criteria formulation (the non-zero weights determine different criteria):

\section{ADJUSTMENT CRITERIA FORMULATION:}

- Formulation 1:

$$
\min \mathrm{G}_{1}\left(X^{t}\right)=\pi_{11} F_{1}\left(A_{X^{t}}\right)+\pi_{12} F_{2}\left(A_{X^{t}}\right)+\pi_{13} F_{1}\left(B_{X^{t}}\right)+\pi_{14} F_{3}\left(B_{X^{t}}\right)
$$

- Formulation 2:

$$
\min \mathrm{G}_{2}\left(X^{t}\right)=\pi_{21} F_{1}\left(A_{X^{t}}\right)+\pi_{22} F_{4}\left(A_{X^{t}}\right)+\pi_{23} F_{1}\left(B_{X^{t}}\right)+\pi_{24} F_{5}\left(B_{X^{t}}\right)
$$

- Formulation 3:

$$
\min \mathrm{G}_{3}\left(X^{t}\right)=\pi_{31} F_{1}\left(A_{X^{t}}\right)+\pi_{32} F_{6}\left(A_{X^{t}}\right)+\pi_{33} F_{1}\left(B_{X^{t}}\right)+\pi_{34} F_{6}\left(B_{X^{t}}\right) .
$$

Note that formulation 1 with $\pi_{13}=1$ and $\pi_{1 k}=0, \forall k \neq 3$ corresponds to the method of Matuszewski, Pitts and Sawyer (1964) for the column coefficient estimation problem. 
The adjustment problem is

$$
\min \mathrm{G}(X) \text { subject to } X \in \chi
$$

where $\mathrm{G}=\mathrm{G}_{\mathrm{i}}$ for some $i$ and certain weights $\pi_{i k}$, and $\chi$ is the feasible set defined by certain constraints on $X$. These constraints can be formulated as:

$$
\begin{aligned}
& \rho_{i 1} x_{i 1}+\rho_{i 2} x_{i 2}+\ldots+\rho_{i n} x_{i n}=(\leq)(\geq) \alpha_{i} \\
& \gamma_{1 j} x_{1 j}+\gamma_{2 j} x_{2 j}+\ldots+\gamma_{n j} x_{n j}=(\leq)(\geq) \beta_{j}
\end{aligned}
$$

The problems solved here only consider the column coefficients, due to their importance in IO analysis. They try to achieve a more homogeneous adjustment of these coefficients by adding new objectives to the ones used by Matuszewski, Pitts and Sawyer (1964). These new objectives aim to reduce the disparities in the relative changes of the coefficients in each of the columns. The new previously mentioned adjustment criteria generate the following problems:

Problem 1: This problem, called DESV1, is defined by $\pi_{11}=\pi_{12}=0, \pi_{13}=\pi_{14}=1$ and constraints (2) and (3). In this case the new element of the objective function tries to minimize the dispersion of the relative changes of each column coefficient with respect to the average relative change of all the elements of its column.

$$
\min \sum_{(\mathrm{i}, \mathrm{j}) \in S_{x}}\left|\frac{b_{i j}^{t}-b_{i j}^{0}}{b_{i j}^{0}}\right|+\sum_{(i, j) \in S_{x}}\left|\frac{b_{i j}^{t}}{b_{i j}^{0}}-v_{j}\right|
$$

subject to

$$
\begin{gathered}
v_{j}=\frac{1}{n_{j}} \sum_{i \in I_{j}} \frac{b_{i j}^{t}}{b_{i j}^{0}} \\
\sum_{j} b_{i j}^{t} \gamma_{j}=\gamma_{i} \quad \forall i \in I \\
\sum_{i} b_{i j}^{t}=1 \quad \forall j \in J
\end{gathered}
$$

This problem can be formulated as the following linear program 


$$
\min \sum_{(\mathrm{i}, \mathrm{j}) \in S_{x}}\left(y_{i j}+z_{i j}+u_{i j}+v_{i j}\right)
$$

subject to

$$
\begin{aligned}
& \frac{b_{i j}^{t}-b_{i j}^{0}}{b_{i j}^{0}}=y_{i j}-z_{i j} \quad \forall(i, j) \in S_{X} \\
& \frac{b_{i j}^{t}}{b_{i j}^{0}}-v_{j}=u_{i j}-v_{i j} \quad \forall(i, j) \in S_{X} \\
& v_{j}=\frac{1}{n_{j}} \sum_{i \in I_{j}} \frac{b_{i j}^{t}}{b_{i j}^{0}} \\
& \sum_{j \in J_{i}} b_{i j}^{t} \gamma_{j}=\gamma_{i} \quad \forall i \in I \\
& \sum_{i \in I_{j}} b_{i j}^{t}=1 \quad \forall j \in J \\
& y_{i j}, z_{i j}, u_{i j}, v_{i j} \geq 0 \quad \forall(\mathrm{i}, \mathrm{j}) \in S_{X}
\end{aligned}
$$

Problem 2: This problem, called DESV2, is defined by $\pi_{21}=\pi_{22}=0, \pi_{23}=\pi_{24}=1$ and constraints (2) and (3). It uses the variables that capture the absolute value of the relative changes, in order to reduce the differences in the column coefficient relative changes between contiguous rows.

$$
\begin{gathered}
\min \sum_{(\mathrm{i}, \mathrm{j}) \in S_{x}}\left|\frac{b_{i j}^{t}-b_{i j}^{0}}{b_{i j}^{0}}\right|+\sum_{(i, j) \in(I-\{m\}) \times J}|| d_{i j}-1|-| d_{i+1 j}-1 \mid \\
d_{i j}=\left\{\begin{array}{ccc}
\frac{b_{i j}^{t}}{b_{i j}^{0}} & \text { if } & (i, j) \in S_{x} \\
1 & \text { otherwise }
\end{array}\right.
\end{gathered}
$$

subject to

$$
\begin{array}{ll}
\sum_{j} b_{i j}^{t} \gamma_{j}=\gamma_{i} & \forall i \in I \\
\sum_{i} b_{i j}^{t}=1 & \forall j \in J
\end{array}
$$

This problem can be formulated as the following linear problem 


$$
\min \sum_{(i, j) \in S_{X}}\left(y_{i j}+z_{i j}+u_{i j}+v_{i j}\right)
$$

subject to

$$
\begin{gathered}
\frac{b_{i j}^{t}-b_{i j}^{0}}{b_{i j}^{0}}=y_{i j}-z_{i j} \quad \forall(i, j) \in S_{X} \\
\left(y_{i j}+z_{i j}\right)-\left(y_{i+1 j}+z_{i+1 j}\right)=u_{i j}-v_{i j} \quad \forall(\mathrm{i}, \mathrm{j}) \in(I-\{m\}) \times J \\
y_{i j}=z_{i j}=0 \quad \forall(\mathrm{i}, \mathrm{j}) \notin S_{X} \\
y_{i j}, z_{i j}, u_{i j}, v_{i j} \geq 0 \quad \forall(\mathrm{i}, \mathrm{j}) \in S_{X} \\
\sum_{j} b_{i j}^{t} \gamma_{j}=\gamma_{i} \quad \forall i \in I \\
\sum_{i} b_{i j}^{t}=1 \quad \forall j \in J
\end{gathered}
$$

Problem 3: This problem, called MMAX, is defined by $\pi_{31}=\pi_{32}=0, \pi_{33}=1$ and $\pi_{34}=\pi$, where $\pi$ is the cardinal of $S_{X}$, and constraints (2) and (3). This problem includes a minimax criterium in order to minimize the maximum relative change among the elements of each column.

$$
\min \sum_{(\mathrm{i}, \mathrm{j}) \in S_{X}}\left|\frac{b_{i j}^{t}-b_{i j}^{0}}{b_{i j}^{0}}\right|+\pi \max _{(i, j) \in S_{X}}\left|\frac{b_{i j}^{t}-b_{i j}^{0}}{b_{i j}^{0}}\right|
$$

subject to

$$
\begin{array}{ll}
\sum_{j} b_{i j}^{t} \gamma_{j}=\gamma_{i} & \forall i \in I \\
\sum_{i} b_{i j}^{t}=1 & \forall j \in J
\end{array}
$$

This problem can be formulated as the following linear problem

$$
\min \sum_{(i, j) \in S_{X}}\left(y_{i j}+z_{i j}\right)+\pi u
$$

subject to

$$
\frac{b_{i j}^{t}-b_{i j}^{0}}{b_{i j}^{0}}=y_{i j}-z_{i j} \quad \forall(i, j) \in S_{X}
$$




$$
\begin{array}{ll}
y_{i j}+z_{i j} \leq u & \forall(\mathrm{i}, \mathrm{j}) \in S_{X} \\
\sum_{j} b_{i j}^{t} \gamma_{j}=\gamma_{i} & \forall i \in I \\
\sum_{i} b_{i j}^{t}=1 & \forall j \in J \\
y_{i j}, z_{i j} \geq 0 & \forall(\mathrm{i}, \mathrm{j}) \in S_{X}
\end{array}
$$

Other problems could obviously be formulated as combinations of these three.

\subsection{Analysis of the models proposed}

In this section we proceed to present and analyze the results of the different comparisons prepared to measure the usefulness of the models proposed. All the applications presented in this work used the SAM of Mozambique prepared by Robinson, S. Cattaneo, A. and El-Said, M (2001). All the models have been solved combining the optimization and computational capabilities of GAMS and MATLAB respectively, using the link developed by Michael C. Ferris (1999).

The cases prepared correspond to problems 1 to 3 in the previous section, and are compared with the RAS and MSCE methods. Using the SAM of Mozambique as a starting point, we generated new SAMs not allowing for a decrease in the different row and column totals and imposing the value of the maximum increase for these values. These upper limits ranged between $10 \%$ and $100 \%$, while the lower limit was always $0 \%$. For each of these ranges the number of problems generated was 3000 . For each of these 3000 cases the five adjustment criteria (MSCE, RAS, DESV1, DESV2 and MMAX) were solved. We therefore obtained 3000 column coefficients matrices for each method, totaling 15000 matrices for each upper limit. 
The following measures have been used in order to compare the coefficients obtained from each of these 15000 new column coefficient matrices with the original coefficient matrix:

1. Mean absolute difference:

$$
\operatorname{MAD}\left(X^{0}, X\right)=\frac{\sum_{(i, j)}\left|x_{i j}-x_{i j}^{0}\right|}{m \times n}
$$

2. Mean relative difference:

$$
\operatorname{MRD}\left(X^{0}, X\right)=\frac{\sum_{(i, j) \in s_{x}^{0}}\left|\frac{x_{i j}-x_{i j}^{0}}{x_{i j}^{0}}\right|}{m \times n}
$$

3. Maximal absolute difference:

$$
\operatorname{MXAD}\left(X^{0}, X\right)=\max _{(i, j)}\left|x_{i j}-x_{i j}^{0}\right|
$$

4. Maximal relative difference:

$$
\operatorname{MXRD}\left(X^{0}, X\right)=\max _{(i, j) \in S_{X^{0}}}\left|\frac{x_{i j}-x_{i j}^{0}}{x_{i j}^{0}}\right|
$$

For each of these 15000 new column coefficient matrices associated with each upper limit, these six measures have been used to compare the three methods proposed (DESV1, DESV2 and MMAX) with MSCE and RAS. This comparison was done calculating the ratio between both groups of values, thus obtaining six groups of 3000 ratios (DESV1/MSCE, DESV2/MSCE, MMAX/MSCE, DESV1/RAS, DESV2/RAS, MMAX/RAS) for each comparison measure and upper limit.

Assuming each set of 3000 ratios -obtained for each ratio, upper limit and measureconstitute a normal distribution sample, the interval containing the mean of the distribution, with a 0.05 significance level, was calculated. In all cases, both interval limits were located on the same side of the value 1 . 
Table 1 shows the results obtained by the comparisons carried out between all these methods showing the upper limit of the mentioned interval. This upper limit corresponds with the worst value for our proposed adjustment methods.

For example, the value 0.771 located in the column corresponding to the 3000 SAMs generated with a $30 \%$ maximum increase in the value of the column and row totals, and the row showing the ratio of the mean relative differences between DESV1 and MSCE, is the upper limit of the interval of this ratio. This shows that the mean relative difference obtained with our method DESV1 is at least a $32,86 \%$ lower than the same comparison criteria obtained with the MSCE method. 
Table 1

\section{Results obtained with the proposed methods}

\begin{tabular}{|c|c|c|c|c|c|c|c|}
\hline & $100 \%$ & $75 \%$ & $50 \%$ & $40 \%$ & $30 \%$ & $20 \%$ & $10 \%$ \\
\hline \multicolumn{8}{|c|}{ Maximum absolute difference } \\
\hline desv $1 / \mathrm{msce}$ & 1.261 & 1.359 & 1.577 & 1.733 & 1.920 & 2.154 & 2.413 \\
\hline desv $2 / \mathrm{msce}$ & 1.277 & 1.367 & 1.580 & 1.743 & 1.926 & 2.166 & 2.429 \\
\hline $\mathrm{mmax} / \mathrm{msce}$ & 1.297 & 1.383 & 1.563 & 1.722 & 1.839 & 1.995 & 2.142 \\
\hline desv1/ras & 1.112 & 1.135 & 1.221 & 1.292 & 1.360 & 1.448 & 1.567 \\
\hline desv2/ras & 1.129 & 1.145 & 1.227 & 1.307 & 1.370 & 1.465 & 1.588 \\
\hline $\operatorname{mmax} / \mathrm{ras}$ & 1.145 & 1.157 & 1.214 & 1.297 & 1.314 & 1.352 & 1.395 \\
\hline \multicolumn{8}{|c|}{ Mean absolute difference } \\
\hline desv $1 / \mathrm{msce}$ & 1.311 & 1.320 & 1.359 & 1.253 & 1.275 & 1.303 & 1.353 \\
\hline desv $2 / \mathrm{msce}$ & 1.350 & 1.342 & 1.368 & 1.289 & 1.301 & 1.318 & 1.372 \\
\hline $\operatorname{mmax} / \mathrm{msce}$ & 1.472 & 1.481 & 1.510 & 1.464 & 1.428 & 1.391 & 1.395 \\
\hline desv1/ras & 1.153 & 1.121 & 1.116 & 0.987 & 0.986 & 0.984 & 1.003 \\
\hline desv2/ras & 1.194 & 1.143 & 1.125 & 1.021 & 1.011 & 0.996 & 1.018 \\
\hline $\operatorname{mmax} / \mathrm{ras}$ & 1.278 & 1.236 & 1.219 & 1.157 & 1.110 & 1.054 & 1.033 \\
\hline \multicolumn{8}{|c|}{ Maximum relative difference } \\
\hline desv $1 / \mathrm{msce}$ & 0.982 & 0.962 & 0.938 & 0.972 & 0.940 & 0.935 & 0.979 \\
\hline desv $2 / \mathrm{msce}$ & 1.723 & 1.442 & 1.200 & 0.979 & 0.945 & 0.946 & 1.021 \\
\hline $\operatorname{mmax} / \mathrm{msce}$ & 0.858 & 0.768 & 0.696 & 0.706 & 0.625 & 0.524 & 0.435 \\
\hline desv1/ras & 1.103 & 1.092 & 1.099 & 1.140 & 1.142 & 1.194 & 1.345 \\
\hline desv2/ras & 1.861 & 1.694 & 1.361 & 1.144 & 1.155 & 1.215 & 1.411 \\
\hline $\operatorname{mmax} / \mathrm{ras}$ & 0.945 & 0.847 & 0.786 & 0.791 & 0.718 & 0.637 & 0.587 \\
\hline \multicolumn{8}{|c|}{ Mean Relative difference } \\
\hline desv $1 / \mathrm{msce}$ & 1.044 & 1.000 & 0.939 & 0.826 & 0.771 & 0.690 & 0.579 \\
\hline desv $2 / \mathrm{msce}$ & 1.415 & 1.261 & 1.110 & 0.835 & 0.789 & 0.721 & 0.637 \\
\hline $\operatorname{mmax} / \mathrm{msce}$ & 1.473 & 1.371 & 1.280 & 1.026 & 0.959 & 0.879 & 0.815 \\
\hline desv1/ras & 1.015 & 0.937 & 0.867 & 0.749 & 0.707 & 0.637 & 0.545 \\
\hline desv2/ras & 1.403 & 1.199 & 1.035 & 0.756 & 0.722 & 0.667 & 0.601 \\
\hline $\operatorname{mmax} / \mathrm{ras}$ & 1.434 & 1.281 & 1.178 & 0.923 & 0.874 & 0.811 & 0.769 \\
\hline
\end{tabular}

Note: $\mathrm{msce}=$ minimum sum of cross entropies; desv1=first method proposed (average relative change by column); desv2= second method proposed (relative changes between contiguous rows); $\operatorname{mmax}=$ third method proposed (minimax criterium). 
If we take the first two measures into account, the maximum and mean absolute difference, Table 1 shows worse results for the methods we propose. This is a reasonable result, since our methods try to minimize the relative differences between the column coefficients instead of the absolute ones. We can observe that the results obtained by our methods are closer to the ones obtained for RAS than the ones reached by the MSCE method. This is actually also the case for the rest of the measures considered. If we consider the maximum absolute difference separately, our three methods show a similar behavior. They start with large ratios, but they show a clear declining path as soon as we increase the changes in the row and column totals.

However, for the mean absolute difference, no uniform tendency can be observed. In the comparisons with the MSCE method the ratios ameliorate up to and including $40 \%$ changes in the row and column totals, except for the MMAX method. If we observe the ratios for the comparisons with the RAS method, the tendencies are more disparate. Again, the differences between RAS and our methods are very small, specially for the range between $10 \%$ and $40 \%$.

If we now consider the measures of maximum and mean relative differences, our methods proposed always show better results, for the range of changes between $10 \%$ and $40 \%$, with the exception of the ratios relating to the first two methods proposed (DESV1 and DESV2) with respect to RAS for the maximum relative difference, and the ratio comparing the MMAX method with the MSCE for the mean relative difference and the $40 \%$ interval. Moreover, for the maximum relative difference, the MMAX always shows better results compared to both the MSCE and the RAS methods. The DESV1 and DESV2 methods always show better values only when compared to the MSCE method.

\section{4.- Conclusions}

This work is based on the conviction that any updating process should try to minimize the relative deviation of the new coefficients from the initial ones in a homogeneous way. This homogeneity means for us that the magnitude of this relative deviation should be as similar as possible among the elements of each row or column. Therefore, any 
measure of the degree of homogeneity should be defined in terms of relative differences.

In this paper, we have compared the ability of different adjustment methods to preserve the structure of the original column coefficient matrix in a homogeneous way. In order to carry out this objective we have used the maximum and the mean relative difference comparison measures. However, we also dealt with other comparison indicators to broaden the scope of the study.

Our results clearly show the fact that, for deviations equal to or less than $40 \%$ between the row and column totals of the original and he final SAMs, the methods we propose DESV1, DESV2 and MMAX- allow us to obtain a more homogeneous adjustment than with the other two methods taken into account, RAS and MSCE. We should take into consideration that these better results in terms of homogeneity -lower maximum and mean relative differences- are accompanied by very similar results, in terms of the mean absolute difference, to the ones achieved by RAS. Therefore, we have been able not only to obtain a more homogeneous adjustment for an important range of cases, but also to keep the mean of the absolute changes near to the ones observed by the RAS method.

Should we not feel very satisfied with the results in terms of the mean and maximum absolute differences, we should bear in mind that our proposals could be very easily modified to combine the use of both absolute and relative measures. This could be done either by imposing limits to the maximum changes in the absolute differences or by incorporating new elements in our objective function that allow us to weight differently the absolute and relative differences in the coefficients, turning the problem into a multiobjective one.

The methods we propose are especially suited for cases in which the row and column totals of the new SAM diverge within the indicated limits. We should however take into account that any of the considered adjustment processes can be separated into two stages. The first stage could consist of allowing the total of the columns and rows of our SAM to change in the same proportion, which would correspond to the minimum of all the changes envisaged. The problem is a trivial one since the new column coefficients 
would be exactly equal to the coefficients of the original matrix. The next stage would adjust the SAM obtained in the previous stage in order to obtain the final SAM. The changes in the total column and row sums involved in this second stage are necessarily smaller than the ones needed to be considered should we omit the first stage. This procedure allows for the increase in the number of situations where our methods can be considered to be more effective.

Since our main objective consists of maintaining the relative structure of the coefficients as stable as possible, the methods we propose should also be mainly applied to situations where this behavior makes economic sense. This could be the case of those National Statistics Institutes that use IO tables increasingly for their yearly national accounts calculations. The results we present in this paper, could be of some help to those in charge of these efforts and, in general, to anyone that needs to update a SAM or IO table without incorporating any structural change in the coefficients. They can also be easily adapted to the AUP of Input-Output Tables. 


\section{References}

Bacharach (1965): "Estimating Nonnegative Matrices from Marginal Data", International Economic Review, Vol. 6. №3, 294 - 310

Ferris, Michael C., (1999): "Matlab and Gams: Interfacing Optimization and Visualization Software”. http://www.cs.wisc.edu/math-prog/matlab.html

Golan, Judge and Miller, (1996); "Maximum Entropy Econometrics, Robust Estimation with Limited Data”. (John Wiley \& Sons)

Golan, Judge and Robinson, (1994): "Recovering Information from Incomplete or Partial Multisectoral Economic Data", The Review of Economics and Statistics 76, 541 $-9$

Harrigan, F.J. and Buchanan, I., (1984): “A Quadratic Programming Approach to IO Estimation and Simulation", Journal of Regional Science, Vol. 24, 3, 339 - 358

Macgill, S.M., (1977): “Theoretical properties of biproportional matrix adjustment", Environment and Planning A, Vol. 9, 687 - 701

Matuszewski T.I., Pitts P.R. and Sawyer J.A., (1964): "Linear Programming Estimates of Changes in Input Coefficients", Canadian Journal of Economics and Political Science, Vol. XXX,2, 203 - 211

McDougall, (1999): "Entropy Theory and RAS are Friends" http://www.sjfi.dk/gtap/papers/McDougall.pdf

Robinson, S. Cattaneo, A. and El-Said, M, (2001): "Updating and Estimating a Social Accounting Matrix Using Cross Entrophy Mehods", Economic Systems Research. Vol. 13. No. $1.47-64$ 
Schneider and Zenios, (1990): "A Comparative Study of Algorithms for Matrix Balancing”, Operations Research. Vol. 38. No. 3.

Stone, R. and J.A.C. Brown, A. (1962): “A computable Model of Economic Growth”. (A Programme for Growth 1), (Chapman and Hall, London)

Zenios, S.A., Drud, A. and Mulvay, J. ,(1989): "Balancing large Social Accounting Matrices with Nonlinear Network Programming”, Networks, Vol. 19569 - 5857 